\title{
ENSEÑANZA DE LA BIOÉTICA EN EL CURRÍCULO DE LAS CARRERAS DE ODONTOLOGÍA DESDE LA PERSPECTIVA DE LOS ESTUDIANTES
}

\author{
Carlos Zaror Sánchez¹, Patricia Muñoz Millán², Gerardo Espinoza Espinoza², Carolina Vergara Gon- \\ zález ${ }^{3}$ Patricio Valdés García ${ }^{1}$
}

Resumen: Se realizó un estudio de tipo descriptivo, a través del análisis de contenido, con la finalidad de conocer las percepciones de los estudiantes de odontología sobre la enseńanza de la bioética en el currículo de la carrera de Odontología de la Universidad de la Frontera. La recolección de los datos se realizó mediante la técnica de grupos focales, en dos sesiones de doce alumnos cada uno, representados por dos alumnos de los diferentes niveles de la carrera. La interrelación entre los participantes se estimuló a través de una pauta de conducción previamente diseñada. Los datos, registrados a través de grabaciones, fueron transcritos para el análisis de contenido, efectuado manualmente mediante triangulación entre los investigadores. A partir del análisis de contenido de los datos primarios, se estableció una serie de categorías, agrupadas en cuatro grandes dimensiones: concepto de bioética, importancia de la bioética en el ejercicio profesional, enseñanza-aprendizaje de la bioética y modalidad de enseńanza de la bioética. Los estudiantes de odontología de la Universidad de la Frontera transitan de manera muy asertiva por los tópicos que componen la compleja dimensión de la enseñanza de la bioética y reconocen la importancia de la bioética en su formación.

Palabras clave: ética, bioética, enseñanza, odontología, currículo

\section{Training on bioethics in dentistry curriculum from the perspective of students}

\begin{abstract}
A descriptive study was carried out through content analysis, with the goal of knowing the perceptions of dentistry students about bioethics training in Dentistry curriculum of Frontera University. Data collected was carried out through focal group technique in two sessions of 12 students each, represented by two students from de different levels of the career. The interrelation among participants was stimulated through a guide for conduction previously designed. Data registered through tape recording were transcribed for content analysis, carried out manually by triangulation among researchers. Several categories were established by content analysis of primary data, grouped in four big dimensions: concept of bioethics, importance of bioethics in professional exercise, teaching-learning of bioethics and modality of teaching of bioethics. Dentistry students of Frontera University walked in assertive way through issues belonging to the complex dimension of bioethics training and recognize the importance of bioethics in their training.
\end{abstract}

Key words: ethics, bioethics, teaching, dentistry, curriculum

\section{Ensino da bioética no currículo dos cursos de odontologia sob a perspectiva dos estudantes}

Resumo: Foi realizado um estudo de tipo descritivo, através da análise de conteúdo, com a finalidade de conhecer as percepçôes dos estudantes de odontologia sobre o ensino da bioética no currículo do curso de Odontologia da Universidad de la Frontera. A coleta dos dados foi realizada mediante a técnica de grupos focais, nas seçóes de doze alunos cada uma, representados por dois alunos dos diferentes níveis do curso. A inter-relação entre os participantes foi estimulada por meio de uma pauta de condução previamente projetada. Os dados, registrados através de gravaçôes, foram transcritos para a análise de conteúdo, efetuado manualmente mediante triangulaçáo entre os investigadores. A partir da análise de conteúdo dos dados primários, se estabeleceu uma série de categorias, agrupadas em quatro grandes dimensóes: conceito de bioética, importância da bioética no exercício profissional, ensino-aprendizagem da bioética e modalidade de ensino da bioética. Os estudantes de odontologia da Universidad de la Frontera transitam de maneira muito assertiva pelos tópicos que compóem a complexa dimensão do ensino da bioética e reconhecem a importância da bioética em sua formação.

Palavras-chave: ética, bioética, ensino, odontologia, currículo

\footnotetext{
${ }^{1}$ Programa de Especialización en Odontopediatria, Universidad de Frontera, Temuco, Chile Correspondencia: czaror@ufro.cl

${ }^{2}$ Departamento de Salud Pública, Facultad de Medicina, Universidad de Frontera, Temuco, Chile

${ }^{3}$ Departamento de Odontología Integral, Facultad de Medicina, Universidad de la Frontera, Temuco, Chile
} 


\section{Introducción}

Como en otras disciplinas del área de la salud, la bioética forma parte integral de la práctica diaria en la profesión odontológica; por esto, su incorporación en la malla curricular de esta carrera es indispensable para preparar al estudiante en la toma de decisiones y resolución de dilemas éticos propios del ejercicio profesional. Además, la formación universitaria es una instancia propicia para alcanzar niveles superiores de desarrollo moral, logrando modelos de conducta ética que se aplicarán en la vida adulta, tanto en el contexto personal como en el ámbito profesional $(1,2)$.

La enseñanza de la ética en la universidad ha evolucionado tanto como lo han hecho las tendencias educativas a través de la historia. En un comienzo, esta disciplina fue enseñada en combinación con cursos de áreas afines, como: historia dental, gestión, jurisprudencia, deontología, medicina legal, etc. Estos cursos estaban basados en metodologías tradicionales de enseñanza-aprendizaje que no promovían el diálogo entre los estudiantes y sus formadores, por lo que el grado de motivación y conocimientos logrados eran muy escasos(3-5). Actualmente, en sintonía con el Aprendizaje Centrado en el Estudiante, son las estrategias participativas y vivenciales las más utilizadas para la entrega del componente ético en la formación universitaria $(6,7)$. La finalidad de estos cambios es que los dilemas éticos sean abordados en el momento en que se van presentando de forma natural. Bajo este contexto, en la literatura científica disponible existe un amplio consenso que avala el hecho de que la ética debe ser enseñada de forma multidisciplinaria e interprofesional $(8-10)$.

La principal metodología usada en los nuevos currículos es el aprendizaje basado en problemas $(\mathrm{ABP})$. Esta técnica favorece el desarrollo de habilidades y actitudes en relación con temas éticos, estimula el trabajo en equipo y la capacidad de escuchar otras opiniones. Por otro lado, facilita el razonamiento ético y la toma de decisiones por parte del estudiante (5,10-12). El aprendizaje basado en casos reales también está documentado en la literatura, como una manera de capturar la atención de los estudiantes y hacer la instrucción de la ética clínicamente relevante y motivado$\mathrm{ra}(8,13)$.
Entendiendo que, dentro de este nuevo contexto educativo, son los estudiantes los protagonistas de su propio aprendizaje, conocer su percepción sobre la importancia de la incorporación de la bioética en la malla curricular, así como las estrategias de su enseñanza, es un elemento fundamental para implementar cambios e innovaciones al respecto. Por tal razón, el objetivo de esta investigación es conocer la importancia de la enseñanza de la bioética, desde la perspectiva de los alumnos, en la Carrera de Odontología de la Universidad de la Frontera.

\section{Materiales y método}

\section{Tipo y diseño}

Se realizó un estudio cualitativo, descriptivo, a través del análisis de contenido, utilizando grupos focales como estrategia de recolección de la información.

\section{Población de estudio}

El universo del estudio correspondió a los estudiantes de la carrera de Odontología, Facultad de Medicina, Universidad de la Frontera, de primero a sexto año.

\section{Selección de la muestra}

Los participantes fueron escogidos dentro de los diferentes niveles de la carrera. Los criterios de inclusión fueron: estudiantes ingresados a la carrera entre 2000 y 2010, igual proporción de hombres y mujeres, y voluntad de participar en el grupo focal expresada a través de la firma de un consentimiento informado. Se excluyó a los alumnos(as) sancionados por problemas éticos o que tuvieran alguna profesión previa.

Se decidió hacer una muestra intencionada, con el fin de recolectar información de aquellos alumnos que estaba dispuestos a dar su opinión y que eran visualizados de esta manera por sus docentes y por sus pares.

Para hacer la primera selección de participantes se realizó un sondeo previo, con la finalidad de buscar alumnos que tuvieran algún liderazgo dentro de su nivel. La muestra intencionada se efectuó entre los docentes de la carrera que conocieran los 
respectivos cursos, pidiendo indicaran a quienes consideraran informantes clave para ser incluidos en el grupo focal. A cada informante clave seleccionado en esta primera etapa se le solicitó que mencionara dos personas que ellos consideraban que representaban la opinión del curso. Cuando los alumnos no aceptaron participar, se seleccionó otro alumno de acuerdo al orden de la lista de informante claves.

Los alumnos seleccionados fueron invitados a participar directamente por el equipo de investigación, momento en el cual se les explicó el propósito del estudio.

\section{Técnica de recolección de datos}

La sesión del grupo focal se realizó con la participación de doce alumnos de odontología por grupo, quienes evaluaron la importancia de la bioética en el currículo de la carrera y cómo, a juicio de ellos, esta debe ser enseñada.

La moderación de los grupos estuvo a cargo de uno de los investigadores, con una duración aproximada de 60 minutos.

Se les solicitó a los participantes su consentimiento para grabar la sesión, asegurando la confidencialidad de sus dichos y el anonimato de los participantes. Un segundo investigador se encargó de vigilar la grabación, tomar nota de datos importantes y/o relevantes de la discusión, y registrar observaciones sobre mensajes no verbales concernientes a la discusión.

La interrelación entre los participantes se estimuló a través de una pauta de conducción del grupo focal (pauta 1).

Pauta 1. Pauta de conducción grupo focal

1. ¿Qué entienden ustedes por bioética?

¿Qué diferencia reconocen entre bioética y ética?

2. ¿Cuál es la importancia que ustedes otorgan a la bioética en el ejercicio profesional de la odontología?

¿En qué actividades o situaciones del ejercicio profesional de la odontología consideran ustedes que tiene importancia la bioética?
3. ¿Cuál es la importancia de que a los alumnos de odontología se les incorpore la enseñanza de la bioética en la malla curricular?

4. ¿Cómo se les ha enseñado la bioética hasta ahora?

¿De qué forma consideran ustedes que se debería enseñar la bioética?

\section{Análisis de datos}

Los datos obtenidos a través de las grabaciones fueron transcritos para su análisis de contenido, el que se efectuó de manera manual mediante triangulación entre los investigadores que participaron de la recolección de los datos y aquellos que no lo hicieron.

Usando cada línea de texto como unidad de análisis, se generaron categorías y subcategorías que permitieron la abstracción e integración de los datos. Con el fin de asegurarse de que el análisis reflejaba la opinión de los estudiantes, este fue devuelto a los participantes para sugerencias y aprobación.

\section{Resultados}

A partir del análisis de contenidos de los datos primarios se estableció una serie de categorías, agrupadas en cuatro grandes dimensiones (tabla 1).

Tabla 1. Dimensiones y categorías del análisis de contenido

\begin{tabular}{|l|l|}
\hline DIMENSIÓN & CATEGORÍA \\
\hline & 1. Principios \\
& 2. Normas \\
I. Concepto de & 3. Valores \\
bioética & $\begin{array}{l}\text { 4. Accionar } \\
\text { 5. Relación interpersonal } \\
\text { 6. Derechos de los seres vivos } \\
\text { 7. Campos de la bioética } \\
\text { 8. Reflexión }\end{array}$ \\
\hline \multirow{5}{*}{$\begin{array}{l}\text { II. Importancia de la } \\
\text { bioética en el ejercicio } \\
\text { profesional }\end{array}$} & $\begin{array}{l}\text { 2. Juicio ético ética } \\
\text { 3. Respeto por la dignidad de } \\
\text { los pacientes } \\
\text { 4. Crecimiento profesional } \\
\text { 5. Empatía en la entrega de } \\
\text { información al paciente } \\
\text { 6. Valoración de la decisión del } \\
\text { paciente }\end{array}$ \\
\hline
\end{tabular}




\begin{tabular}{|l|l|}
\hline & $\begin{array}{l}\text { 1. Formación ética familiar } \\
\text { 2. Influencia de la ética } \\
\text { universitaria en la conducta } \\
\text { aprendizaje de la } \\
\text { bioética }\end{array}$ \\
$\begin{array}{l}\text { 3. Nivel de conocimientos de } \\
\text { bioética } \\
\text { 4. Aprendizaje de la ética en la } \\
\text { clínica }\end{array}$ \\
\hline $\begin{array}{l}\text { IV. Modalidad de } \\
\text { enseñanza de la } \\
\text { bioética }\end{array}$ & $\begin{array}{l}\text { 2. ABC } \\
\text { 3. Modelado } \\
\text { 4. Ramo teórico efectuado por } \\
\text { odontólogo } \\
\text { 5. Educación transversal }\end{array}$ \\
\hline
\end{tabular}

Concepto de bioética

Se reconoce que, conceptualmente, la bioética está relacionada con la ética, entendiendo que esta última es más amplia y abarca diferentes ámbitos, y que la bioética está más relacionada con lo biológico o, más especifico aún, con la salud: "La bioética es parte de la ética". "...es como la ética aplicada a la salud".

Otros, con una visión más amplia, consideran a la bioética relacionada con todo lo biológico: "Podría verse relacionada también con esto de los derechos, por ejemplo de los animales, por el concepto 'bio', de biologia y del medio ambiente".

La bioética está relacionada con un conjunto de normas que debieran orientar nuestro actuar: "de las normas que tenemos las personas para obrar bien". Se entiende que los conceptos de "moral" y "bioética" se reflejan en el accionar de las personas: "la moral que está en todos y puede o no llevar a hacer una determinada acción".

Se da mucha importancia a los valores de la persona y a cómo lo que una persona considera valioso puede influir en sus decisiones bioéticas. "Nosotros...vamos a tener que tomar decisiones que afecten tanto al paciente como a nosotros, o a su familia o entorno social, y ahi es donde cumple un rol la ética". "...muchas veces lo que nosotros consideramos correcto, a lo mejor no es lo que el paciente considera correcto".

Los alumnos reconocen, dentro del concepto de "bioética", los cuatro principios definidos por T. L. Beauchamp y J. F. Childress, tanto teóricamente como aplicados a un caso clínico: "La bioética se basa en cuatro principios que son justicia, la autoridad (autonomía), la no maleficencia y la beneficencia". Se observa una confusión al mencionar el principio de autonomía, nombrándolo como principio de autoridad, pero al momento de definirlo se observa que el significado que se le da es el correcto: "en la autoridad va lo que el paciente quiere que le hagan".

En diferentes momentos se refieren a cada uno de ellos. Sobre el de justicia: "Eso, la justicia, porque el paciente no tiene acceso (a la atención de especialista), eso no es justo. El de no maleficencia lo aplican al caso clínico: "...si sabemos que ese diente puede tratarse, le estariamos haciendo un mal al paciente en sacárselo". Respecto de la beneficencia: "tengo que hacerle el bien al paciente". Por ultimo, el de autonomía, que fue sobre el cual más se habló: "hacer esto de la mejor manera posible... respetando los deseos del paciente".

Profundizando sobre los principios bioéticos, debaten respecto de si el principio de autonomía tiene algún límite, por ejemplo, si un paciente les pide hacer algo que no es terapéutico, sino que más bien lo puede dañar. Al final del debate, la opinión que genera consenso es: "Pero ahi es uno...el que tiene que hacer primar la ética de uno mismo". Algunos perciben como más importante el principio de no maleficencia por sobre el de autonomía: "uno no puede hacer algo que le va hacer daño al paciente, en ese caso uno tiene que trasgredir el principio de autonomía". También se da mucho énfasis a que el paciente decida o dé su consentimiento estando debidamente informado: "si uno va ejercer una acción, que el paciente esté informado de lo que se le va hacer".

Se identifican claramente dos campos de acción de la bioética; por un lado, el campo de la salud y, específicamente, la relación odontólogo-paciente, y, por otro, la regulación de las investigaciones con humanos. "Cuando vimos la bioética la vimos bien enfocada a la relación como entre profesionalpaciente"; "creo que en cada uno de nosotros está claro que frente a una persona, frente a un paciente en este caso, frente a un estudio, sabemos que tenemos que aplicar la bioética". 
Importancia de la bioética en el ejercicio profesional

La opinión generalizada y unánime de los estudiantes en torno a este tema es que la bioética es esencial en el ejercicio profesional. Acuden a ejemplos prácticos para destacar su importancia: "La mayoría de los tratamientos que nosotros realizamos, el paciente no los nota, asi que uno puede decir le hice una restauración en tal pieza y en realidad no la necesitaba... ahi hay una falta de ética tremenda".

Visualizan la importancia de la bioética en elementos como el respeto al paciente como ser humano: "Hay que aprender a respetar al paciente, no hay que mentirle al paciente. Eso como que vendría siendo parte de la importancia de la bioética".

También destacan la necesidad de ser empáticos con los pacientes y ponerse en el lugar del otro: "Uno debería tratar de hacer lo mejor para el paciente, tratar de ponerse en el lugar, qué pasa si fuera mi papá, si fuera mi mamá la que se está atendiendo".

Los alumnos también son muy categóricos en afirmar que la bioética en el ejercicio profesional no es circunstancial y que debe aplicarse en todo contexto: "Se relaciona con una forma de ser"; "uno siempre debe hacer lo mejor para el paciente".

Consideran que la bioética es importante para solucionar conflictos que se generan en el quehacer profesional: "Uno trata de hacer lo mejor para el paciente y determina el mejor tratamiento, pero el paciente quiere otra cosa y ahi es donde se genera el conflicto, la bioética está relacionada con eso".

\section{Enseñanza-aprendizaje de la bioética}

Los alumnos consideran que la formación universitaria no condiciona un cambio en la conducta de una persona, ya que las bases de la formación ética se adquieren principalmente al interior de la familia, marcando el actuar moral y ético en las etapas siguientes: "...yo creo que, en realidad, el hecho de tener o no tener un ramo, y no voy a decir que no es importante, pero no creo que vaya a generar mucho cambio en la conducta de la persona, yo creo que ser ético o no es cosa que uno trae desde siempre, desde las bases del hogar, de quien te educó...".
A pesar de lo anterior, los participantes reconocen a la sociedad y a las personas con las que se relacionan, tanto dentro como fuera de la universidad, como elementos importantes en la adquisición de conocimientos y conductas éticas: "...el ser humano se desarrolla en sociedad y según el grupo donde esté también va a tener un poco de ese grupo, entonces, claramente, no depende tanto del ramo, sino de nuestros tutores y como nos vayan amoldando, porque justamente vamos a ser parte de un grupo durante tercero, cuarto, quinto y sexto...".

Parte de la labor educativa de la universidad es la formación ética profesional. Para ello, es necesaria una formación integral en la que los contenidos éticos estén integrados en la enseñanza y en la relación con los pacientes: “...quizás la universidad debe analizar qué tipo de perfil está tratando de crear y cuánto puede influenciar en la transformación de ese perfil...".

En forma recurrente, los participantes mencionan que el sistema de enseńanza-aprendizaje en la clínica odontológica influye en su futuro comportamiento bioético: "En clínica se pone mucho en juego la ética, porque tu tienes que ver un paciente, elegirlo y determinar lo que vas a hacer, y de acuerdo a eso pasas de curso. Yo creo es sumamente importante aplicarla desde que uno es alumno...".

\section{Modalidad de enseñanza de la bioética}

$\mathrm{Al}$ igual que en el ámbito educacional en general, los alumnos demandan estrategias más participativas y vivenciales para la enseñanza de la bioética.

Como se mencionó, las principales metodologías usadas en los nuevos currículos para enseñar la bioética son el aprendizaje basado en problemas y el aprendizaje basado en casos. Estas estrategias de aprendizaje fueron frecuentemente mencionadas por los alumnos como las alternativas más adecuadas. Para ellos, los conceptos y dilemas éticos deberían ser incorporados formalmente en el ramo de Unidad de Integración, el cual utiliza el ABP como metodología de aprendizaje: "Hay un ramo llamado Integración, de primero a tercero, y que dentro de muchos objetivos no estaría nada de mal incorporar, dentro de los objetivos, la aplicación de la bioética". 
Por otro lado, dieron una importancia superlativa al papel que cumple el docente como "modelo" en materia ética. El tutor, con su actuar personal y profesional frente al paciente, puede generar pautas de comportamiento que sirvan de estímulo para sus estudiantes en su proceso de construcción como persona: "...muchas veces la bioética no es necesariamente enseñada como un ramo, o de una forma estructurada, sino que la persona que te está enseñando también te pude ir enseñando ética, y eso es mucho más valioso".

Ellos esperan que todos los docentes, en especial en los ramos clínicos, sirvan de "modelo" frente a los dilemas éticos que se presentan a diario durante la práctica profesional. Por los tanto, los alumnos demandan que los docentes sean individuos responsables, social y profesionalmente, y capacitados formalmente en el área: “...enseñar también un módulo de bioética a todos los docentes, para que una vez que nos enseñen podamos seguir aplicándolo dentro de nuestra formación".

Al referirse a la enseñanza tradicional de la bioética, los alumnos destacaron que un ramo teórico de ética tiene una importancia limitada, al entregar sólo conceptos y directrices generales, pero que no permitiría modificar el razonamiento ético, desarrollar habilidades para el diagnóstico de dilemas éticos y mucho menos lograr cambios conductuales que lleven a una toma de decisión adecuada dentro de un marco social y profesional: "Es que tal vez un ramo no te va a dar la ética que vas a tener tú, pero si las herramientas para aplicarla".

Para ellos es muy importante que el ramo teórico sea enseñando por un odontólogo capacitado en bioética y no por otro profesional: “...un ramo como bioética, si te la enseña un dentista te lo va aplicar a las situaciones clinicas, y entonces te va a servir y vas a pensar —ah me sirvió —".

Todos concordaron que la ética tiene que ser impartida en forma continua, de manera transversal y vertical, a través de los diferentes niveles de la carrera y a medida que los dilemas éticos se vayan presentando: "Yo encuentro que influye mucho más que los docentes nos integren eso desde primero a sexto, en vez de tener un ramo de ética y empezarse a preocupar".

\section{Discusión}

En el ámbito odontológico, a diferencia de otras carreras como medicina y enfermería, la incorporación de esta disciplina al currículo es más reciente y se encuentra en pleno proceso de análisis y evaluación, especialmente en nuestro país. La OPS ha impulsado este proceso desde 2005, a través de la realización de Jornadas de Bioética en Odontología, posicionando definitivamente el tema en el ámbito académico(14).

El concepto de "bioética" ha sufrido una serie de modificaciones desde el momento en que fuera acuñado en la década del setenta por Van Rensselaer Potter. No es de extrañar entonces que los estudiantes no recurran fácilmente a una definición unánime de este concepto, aunque sí conocen el ámbito de su aplicación y desarrollo, recurriendo a ejemplos prácticos para explicarlo. Esta situación también fue estudiada por Buendía(3), quien advierte que el nivel de conocimientos de los alumnos respecto de conceptos bioéticos es bajo, y lo atribuye igualmente al carácter polisémico del término.

Respecto de la enseñanza de la bioética en la educación universitaria, surge como cuestión fundamental considerar o no a la universidad como una instancia propicia para adquirir o modificar actitudes y valores. La opinión de los alumnos en esta materia es que la formación moral está fuertemente determinada por la educación y el ejemplo familiar, y que la etapa universitaria sería complementaria de ésta. Al respecto Quezada(15) reflexiona sobre el papel que desempeña la familia y las instituciones educativas en tal formación. Por un lado, tradicionalmente ha sido el núcleo familiar el encargado de sentar las bases de la conducta del individuo; sin embargo, cada vez más se les exige a las instituciones educativas formar parte en este rol, desde que la dinámica familiar ha cambiado. Por otro lado, como lo explica Román(16), es en la etapa de la adultez — que se vive en la universidad - en la cual el individuo está capacitado para alcanzar niveles superiores de desarrollo moral, logrando reflexionar de manera ética. Tomando en consideración ambos argumentos, queda claro que la formación universitaria en temas bioéticos es fundamental para lograr la formación de profesionales competentes, no sólo 
en ámbito cognoscitivo y procedimental, sino que con la capacidad de enfrentar los dilemas que la profesión demanda y saber tomar decisiones basadas en los saberes éticos(2). Tal afirmación, entonces, genera la necesidad de analizar cómo se incorporará esta disciplina en el currículo. Hay que tener en consideración que deben generarse condiciones para lograr un adecuado ambiente de aprendizaje ético, lo que significa que los contenidos y estrategias, docentes, entre otros aspectos, deben articularse para cumplir tal objetivo.

¿Qué enseñar? y ¿cómo hacerlo? Respecto de la primera pregunta, los estudiantes son claros: no desean teoría, no quieren aprender códigos; insisten en que los contenidos deben ser eminentemente aplicados a las situaciones de la vida profesional y tratados de manera transversal a los largo de la carrera. Diego Gracia(17) propone abordar la enseñanza de pregrado a través de tres pilares: en primer lugar, la ética básica "debe estudiar con una cierta detención y profundidad el tema de los juicios morales y el modo como pueden fundamentarse"; en el período clínico, la ética clínica, que debe ser aplicada, y también la ética profesional o deontología, que debe estar presente antes de terminar la formación profesional. Cardozo y cols. (18) proponen un esquema similar en cuanto a contenidos y también refuerzan la idea de integrar la bioética en asignaturas afines, además de contar con la asignatura propiamente tal, de duración anual o semestral.

Tanto los alumnos como la literatura coinciden en que no se trata de formar expertos en bioética, sino que el modelo ideal supone la integración de contenidos bioéticos en las distintas asignaturas del currículo, entendiendo a la bioética como una transdisciplina(19).

En relación con las estrategias de aprendizaje utilizadas para la enseñanza de la bioética, los alumnos del estudio demandan que estas sean participativas, destacando el APB como un método adecuado para el aprendizaje y con el cual el ya están familiarizados. Diego Gracia(17) propone que sean basadas en el diálogo y la deliberación, puesto que esta estrategia preparará al estudiante para analizar dilemas éticos y generar adecuados procesos de toma de decisiones. Schmidt(20) propone un método de interpretación y análisis holístico de casos bioéticos, que permite el abordaje integral de los problemas desde su identificación hasta la deliberación final. Dada su estructura metódica, resulta ser didáctico y favorece el aprendizaje.

Merece un análisis especial, dentro de las formas de enseñanza/aprendizaje, la función de los docentes, puesto que los estudiantes indican que el profesor como modelo es el principal medio para aprender bioética. Como también lo destacan otros autores, los profesores, para los estudiantes, constituyen un modelo a seguir y la actuación de este logrará influenciarlos, tanto positiva como negativamente(18).

$\mathrm{Al}$ ahondar en el papel del profesor universitario como ente formador ético, es necesario considerar que el docente, como individuo en primer lugar, tiene una moral personal que no puede quedar "fuera de la sala" cuando ejerce su rol como profesor; esto implica entonces que, de manera inevitable, su actividad docente se llevará a cabo desde su propia dimensión moral, desde la que podrá abordar su función educativa en torno a la ética profesional de los estudiantes(16). Una buena estrategia mencionada en la literatura, y también por los alumnos, es la capacitación de los docentes en el área de la bioética, de manera que esta disciplina guíe el quehacer educativo del docente, estrategia que ya ha sido implementada con éxito en otras universidades de Chile(14).

A la luz de estos resultados, surgen nuevas interrogantes en torno a cómo abordar esta problemática. El rol del docente aparece como uno de los elementos fundamentales para el logro del aprendizaje en bioética, pero, ¿qué opinan los propios profesores acerca de esto? Contestar esta pregunta implica involucrar a todos los actores que participan del desafío de formar profesionales del área de la salud y hacerlo con una mirada integradora, considerando la educación en bioética como un pilar fundamental de la educación universitaria. 
Enseñanza de la bioética en odontología desde la perspectiva de los estudiantes - Carlos Zaror Sánchez et al.

\section{Referencias}

1. Bebeau MJ. Can ethics be taught? A look at the evidence: revisited. NY State Dent J 1994; 60(1): 51-57.

2. Martinez M, Buxarrais MR, Esteban F. La universidad como espacio de aprendizaje ético. Revista Iberoamericana de Educación 2002; 29: 17-42.

3. Buendía A, De La Cadena C. Nivel de conocimiento de la bioética en carreras de Odontología de dos Universidades de América Latina. Acta Bioethica 2006; 12(1): 41-47.

4. Miles SH, Lane LW, Bickel J, Walker RM, Cassel CK. Medical ethics education: coming of age. Acad Med 1989; 64(12): 705-714.

5. Siquiera J. Ensino da ética no curso de medicina: a experiencia da Universidade Estadual de Londrina. Bioética 2002; 10(1): 85-95.

6. Aires CP, Hugo FN, Rosalen PL, Marcondes FK. Teaching of bioethics in dental graduate programs in Brazil. Braz Oral Res 2006; 20(4): 285-289.

7. Dávila R. É possível ensinar ética médica em um curso formal curricular? Bioética 2002; 10(1): 115-126.

8. Fox E, Arnold RM, Brody B. Medical ethics education: past, present, and future. Acad Med 1995; 70(9): $761-769$.

9. Goldie J. Review of ethics curricula in undergraduate medical education. Med Educ 2000; 34(2): 108-119.

10. Goldie J, Schwartz L, McConnachie A, Morrison J. Impact of a new course on students' potential behaviour on encountering ethical dilemmas. Med Educ 2001; 35(3): 295-302.

11. De Amorín A, Ferreira E, Freitas R. O lugar da Bioética nos currículos de formacao médica. Rev Bras Educ Med 2006 ;30(2): 56-65.

12. Parker M. Autonomy, problem based-learning, and the teaching of medical ethics. J Med Ethics 1995; $21: 305$.

13. Berk NW. Teaching ethics in dental schools: trends, techniques, and targets. J Dent Educ 2000; 65(8): 744-50.

14. León F. Enseñar Bioética: como trasmitir conocimientos, valores y actitudes. Acta Bioethica 2008; 14(1): 11-18.

15. Quezada A. Hacia de la enseñanza de la Bioética. Acta Bioethica 2008; 14(1): 7-10.

16. Román B. Apuntes para una ética del profesor universitario. Ars Brevis 2001: 355-376.

17. Gracia D. Fundamentación y enseñanza de la bioética. Bogotá: Editorial El Búho;1998:175-183.

18. Cardozo C, Rodriguez E, Lolas F, Quezada A. Ética y odontología. Una introducción. Santiago de Chile: CIEB Universidad de Chile; 2006.

19. Outomuro D. Fundamentación de la enseñanza de la bioética en medicina. Acta Bioethica 2008; 14(1): 19-29.

20. Schmidt L. Método de interpretación y análisis holístico en casos bioéticos. Acta Bioethica 2008; 14(1): 39-46.

Recibido: 21 de enero de 2012

Aceptado: 20 de junio de 2012 